\title{
Noncontact Atomic Force Microscopy Line-Profiling of Irregular Dimers on the Monohydride Si(001) Surface: Tight-Binding Simulation
}

\author{
Akira Masago \\ Graduate School of Engineering Science, Osaka University, \\ 1-3 Machikaneyama, Toyonaka, Osaka 560-8531, Japan
}

(Received 20 May 2013; Accepted 15 June 2013; Published 29 June 2013)

\begin{abstract}
This paper describes curious behaviors in noncontact atomic force microscopy (NC-AFM) line-profiles simulated by the density-functional based tight-binding (DFTB) method. Although NC-AFM observes the surface corrugations in general, these line-profiles show atom-like peaks at vacancy sites. This is caused by a stronger interaction incident to the dangling bond. [DOI: 10.1380/ejssnt.2013.80]
\end{abstract}

Keywords: Ab initio quantum chemical methods and calculations; Surface relaxation and reconstruction; Surface structure and topography; Thermodynamics properties; Silicon; Surface defects

\section{INTRODUCTION}

In the 1990s, NC-AFM was made a contribution to high resolution imaging in a field of the semiconductor science and technology $[1,2]$. Therefore, we currently have a lot of images that are quite clear images as well as complex images that cannot be interpreted easily. In either case, we used to use the computational simulations in order to cultivate a better understanding of the images [3-5]. However, little has been reported for simulations of clear imaging cases compared with the complex cases. The present study shows that the simulations provide us with interesting interpretations in the clear imaging case.

In NC-AFM, corrugations of sample surfaces are imaged with high resolution. On the semiconducting sample surfaces, the NC-AFM images show clear bright spots such as we can regard as individual atoms. Some of the images show bright spots located on the surface atom sites of the energetically stable structures; however, others exhibit the spot pattern that is hardly imagined from the surface atomic configuration $[3,6]$. On a silicon surface on which silver atoms stack, the bright spots did not appear on the surface silver atom sites of the energetically stable structure. Eventually, it is caused due to atom hopping induced by the NC-AFM probe approaching. It was revealed by the numerical simulation. This is just an example, but it is widely known that numerical simulations are a kind of strong tools to provide us with rational interpretations for complex NC-AFM imaging cases.

On the other hand, in the monohydride silicon surface that is one of the clear imaging cases, the numerical simulation has not pursued as deep as the complex cases, because a trivial result has been obtained for the regular surface. In this surface, the experimental images show two types of area, namely regular and irregular. The regular area shows the orderly arrangements of the bright twin spots from which we can easily guess the atomic structure on the surface $[7,8]$. In fact, trivial results were obtained in the simulations: twin bright spots appear on two hydrogen atoms in the energetically stable structure of the monohydride silicon dimer [9-11]. In the irregular area, we see no twin spots, and find that the area is obviously distinct from the regular area. Moreover, it is roughly divided into two types: one is without any spots and the other is with a single spot. If obeying the conclusion of the past paper [9] that the bright spots are on the surface atom sites of the energetically stable structure,
TABLE I: Condition for calculating NC-AFM line-profiles. These values were based on the work of Morita and Sugawara [7].

\begin{tabular}{lccc}
\hline \hline Quantity & Symbol & Value & $(\mathrm{Unit})$ \\
\hline Amplitude & $A_{0}$ & 160.0 & $(\AA)$ \\
Force Constant & $k$ & 4.1 & $(\mathrm{nN})$ \\
Resonance frequency & $\mu_{0}$ & 172.0 & $(\mathrm{kHz})$ \\
Preset frequency shift & $\mu$ & -8.0 & $(\mathrm{~Hz})$ \\
\hline \hline
\end{tabular}

we can naturally guess that hydrogen atoms are leaving the surface depending on the number of the bright spots. Consequently, we expect that the former type is a silicon dimer without any hydrogen atoms, whereas the latter type is a silicon dimer with a single hydrogen atom.

In this paper, the silicon dimer without any hydrogen atoms is called bare dimer, and the silicon dimer with a single hydrogen atom is called one-side-terminated dimer. Besides these two types of defects, dissociation of a silicon dimer itself was also considered. The dissociation of a silicon dimer is known as a familiar defect on the monohydride and clean silicon surfaces, which is traditionally called missing dimer $[12,13]$. In the present simulations, experimental parameters (Table I) are adopted for calculating NC-AFM line-profiles on the constant frequency shift mode, which must be the best condition for detection of the surface hydrogen atoms [7]. Therefore, the missing dimer provided with no peaks on the NC-AFM line-profile, which is a trivial result. In the bare dimer, we guess that it induces no peaks because there is no hydrogen atom on the dimer, but it induces a single peak actually. Lastly, the one-side-terminated dimer induces twin peaks although a single hydrogen atom exists on the dimer, which goes against the expectation. Moreover, the twin peaks are hardly distinguished from the ones of the regular monohydride dimer. These results are seemingly strange, but we find it from the simulation results that they are caused by the relation between the chemical activity on these dimers and the corrugations.

Contents of the present paper are as follows: the second section outlines calculation conditions that are the model structures and calculation methods; the third section provides results and discussion in which activity of the irregular dimers, NC-AFM line-profiles, and the thermal effect are considered; the last section is the summary. 


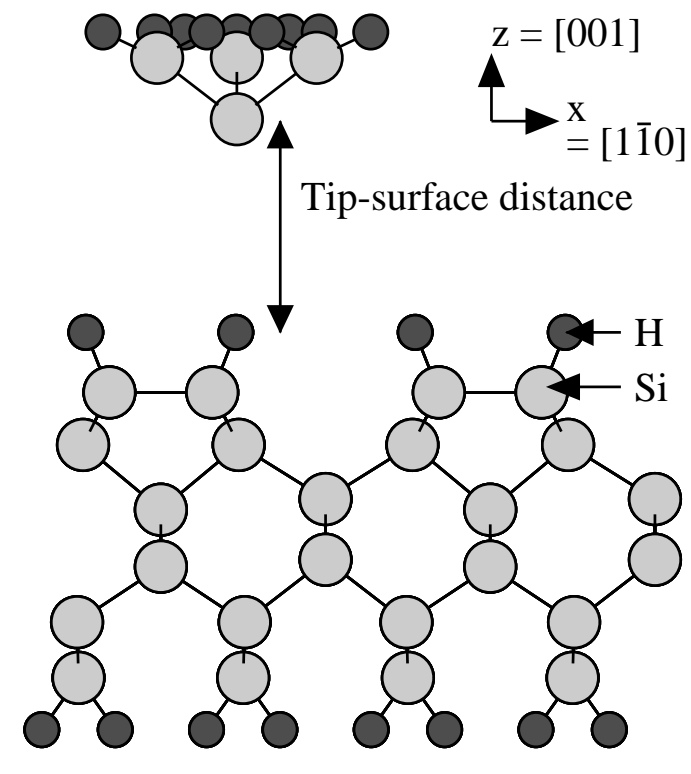

FIG. 1: Model of the regular monohydride $\mathrm{Si}(001)$ surface and the silicon tip. The dark and light gray circles indicate hydrogen and silicon atoms, respectively.

\section{CALCULATION CONDITIONS}

\section{A. Model Structures}

In this study, four $\mathrm{Si}(001)$ monohydride slab models were treated. One of them was a just regular monohydride surface, and the others were irregular monohydride surfaces involving respective irregular dimers.

The regular monohydride surface was prepared as illustrated in Fig. 1. The slab model was set on its (110), (110) and (001) directions along the $\mathrm{x}$-axis, the $\mathrm{y}$-axis, and the $\mathrm{z}$-axis, respectively. Here, the $\mathrm{x}$-axis or the $\mathrm{y}$-axis is often called the lateral direction; the $\mathrm{z}$-axis is called the vertical direction. The slab model consisted of six layers along the vertical direction, and the single layer consisted of $(4 \times 4)$ unit cells along the lateral directions. Here, the periodic boundary condition was applied in the lateral directions. The front surface was a monohydride surface that was the $\mathrm{p}(2 \times 1)$ structure, which had no imperfection. The back surface was terminated by hydrogen atoms, which formed a symmetric dihydride surface.

The other slab models (namely irregular surface models) were prepared from the regular surface model. Each irregular surface model was built by replacing a regular monohydride dimer to an irregular dimer. The first slab structure had a dimer including a dangling bond on itself. That is, a single hydrogen atom connected to one silicon atom of the dimer, and a dangling bond was left on the other silicon atom. As described above, it is called the one-side-terminated dimer in the present paper. The second had a dimer without any hydrogen atoms. It is called the bare dimer here. The last one was the missing dimer. That is, a monohydride dimer was absent from the regular monohydride surface.

Lastly, an NC-AFM tip model was prepared [14, 15]. As the tip model, a $\mathrm{Si}_{4}$ pyramidal cluster was assumed, where the back surface was terminated by nine hydrogen atoms. The tip-surface distance was defined as a vertical length between the apex atom center of the tip cluster and the top-most atom center on the target sample surface (Fig. 1). The distance might change slightly due to the structural optimization, but the effect did not feed back to the tip-sample distance in calculating the frequency shifts.

\section{B. Calculation Methods}

These simulations were performed in accordance with the DFTB method [16, 17]. This method provides us with short-range inter-atomic forces using the HellmannFeynman force method [18]. Besides, the electrostatic interaction between atoms is considered using the Ewald sum [19]. These forces are adopted for calculating forces acting on the tip as well as stable structures. The frequency shift was calculated by the Sasaki-Tsukada method [20], and the parameters were summarized in Table I. The parameters were based on the experimental condition that was the best condition for detection of the surface hydrogen atoms [7].

Moreover, the van der Waals force was considered between the tip and the sample surface via the continuous model suggested by Sasaki and Tsukada [21]. The scheme is independent of the lateral tip position, because they regarded the surface as a plain plate. Therefore, the procedure does not affect a pattern of the simulation images. It just modifies the frequency shifts to the practical values.

\section{RESULTS AND DISCUSSION}

\section{A. Activity of the irregular dimers}

Figure 2 shows the stable surface structures, where all the surfaces were optimized with forces less than $1 \mathrm{pN}$. In the surface including the missing dimer, the stable structure (Fig. 2(A)) shows a significant deformation. The deformation that is new $\mathrm{Si}-\mathrm{Si}$ connections represented by dashed lines are formed at the second layer just below the missing dimer line. The lengths $(2.76 \AA)$ are slightly longer than the length $(2.35 \AA)$ in other reports [12]. However, it is in agreement with them qualitatively, because both the present result and other reports suggest that the new $\mathrm{Si}-\mathrm{Si}$ bonds appear. If these atoms around the missing dimer did not approach each other, the missing dimer site would show strong activity due to dangling bonds left on these atoms. In conclusion, the site must be passive.

In the surface including the one-side-terminated dimer, the stable structure (Fig. 2(B)) shows no deformation except the leaving hydrogen atom, although the other irregular dimers reconstructed as described above and below. Since this behavior leaves the dangling bond as it is, the dimer does not seem to be stabilized. Consequently, the dangling bond site (namely the vacancy site) must show strong activity.

In the surface including the bare dimer, the stable structure (Fig. 2(C)) shows rather deformation. The deformation is a familiar deformation in the $\operatorname{Si}(001)$-c $(4 \times 2)$ surface that is called buckling dimer [22]. It is just the tilting 

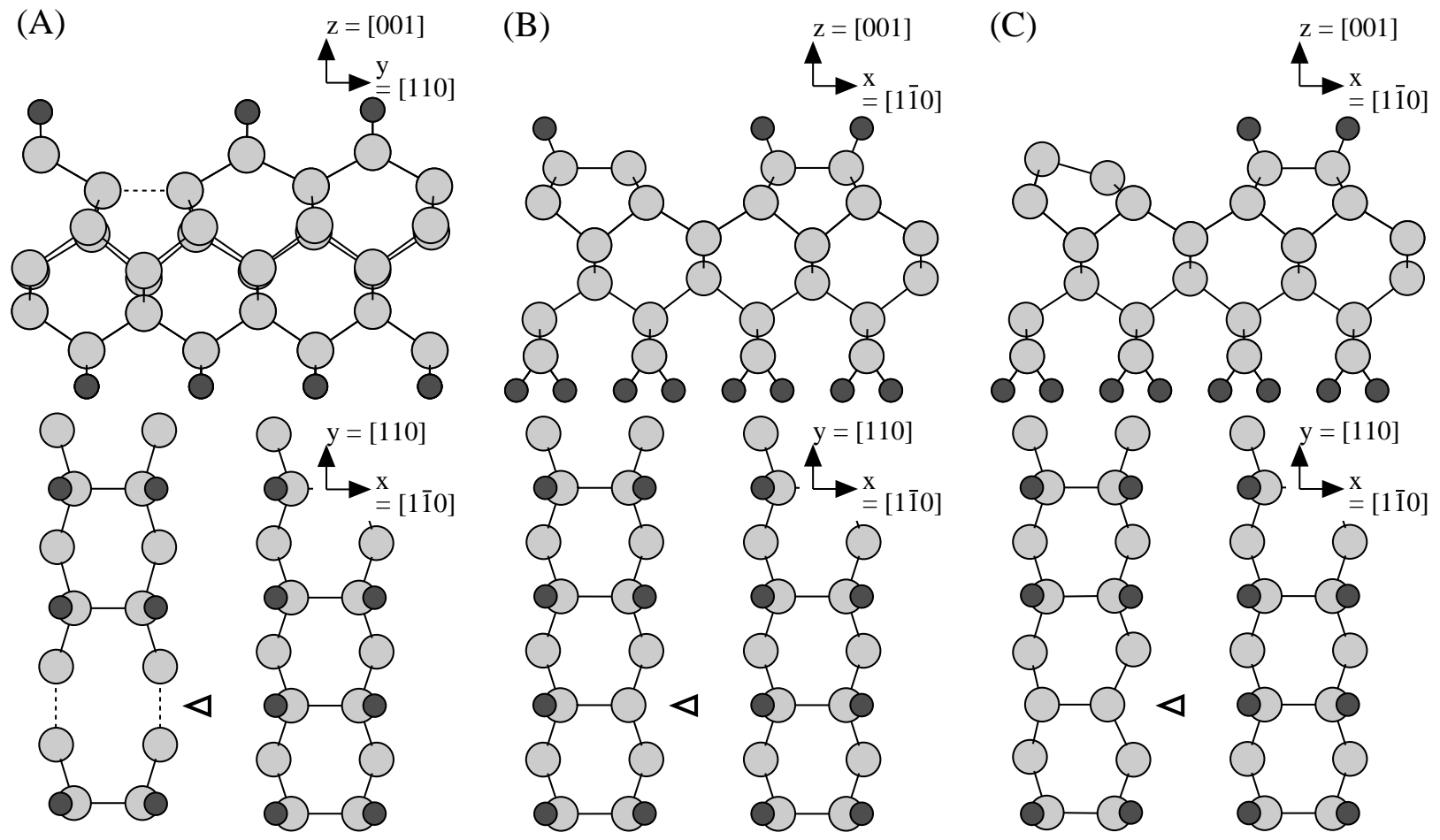

FIG. 2: Side and top views of the optimized defect structures (however, front view for the missing dimer (A)). Missing dimer, one-side-terminated dimer, and bare dimer are shown in (A), (B), and (C), respectively. Each defect site is indicated by an open triangle in the top view.

of the bare dimer, and the tilting angle is 17 degree that almost equals to a typical angle in the $\operatorname{Si}(001)-\mathrm{c}(4 \times 2)$ surface [22]. The tilting (or buckling) makes both the upper and lower atoms stabilize. Thereby the activity of the dangling bonds must be inhibited, although the dangling bonds are left on the dimer.

\section{B. NC-AFM Line-Profiles}

Figures 3(A), 3(B), and 3(C) show NC-AFM lineprofiles on the constant frequency shift mode with the preset frequency shift of $-8.0 \mathrm{~Hz}$ along the missing dimer, the one-side-terminated dimer, and the bare dimer, respectively. Every irregular dimer is drawn together with a regular dimer. For the regular monohydride dimer and the missing dimer $(\mathrm{A})$, the results agree well with the intuitive interpretations. In the regular monohydride dimer, the two atoms each induce the single peaks; consequently, the twin peaks appear on the regular monohydride dimer. The behavior of the regular dimer is the same as the conclusion of the past research. [9] That is, an certain irregular dimer hardly affects the spots of the nearby regular dimer. In the missing dimer (A), no peaks are visible. The line-profile shows simply a deep flat region on the missing dimer. This is because the missing dimer site is passive against the NC-AFM probe, and the behavior coincides with the conclusion in the previous subsection. Besides, the behavior is related to the tip-surface distance. At a height detecting hydrogen atoms, the tip-surface distance is so large that the chemical forces between the tip and the surface go undetected. Finally, this must correspond to no spot area in the experimental images.

In the one-side-terminated dimer (B), we see twin peaks. One is smaller and exists on the hydrogen atom site of the dimer, and the other is taller and at the vacancy site. Thus the pair of spots is asymmetric exactly; however, the height difference is so small that we hardly distinguish from the pair of spots on the regular monohydride silicon dimer that is entirely symmetric. This result is seemingly inconsistent with the previous conclusion: the chemical activity on the vacancy site is strong. However, we find that it is rational by taking the tipsurface distance into consideration: the distance when the tip located on the vacancy site is longer than the distance when the tip located on the atom site. That is, the vacancy site has a strong chemical activity, but the activity cancels out the long tip-surface distance. Therefore, the twin peaks are finally similar to the ones from the regular monohydride silicon dimer.

In the bare dimer $(\mathrm{C})$, a single peak is seen in the lineprofile. This single peak is on the upper atom of the bare dimer, whereas no peak appears on the lower atom. The single peak reflects activity of the upper atom. Here, the activity is smaller than the dangling bond site of the oneside-terminated dimer (B). This is interpreted from the peak height in the bare dimer $(\mathrm{C})$ that is lower than the one in (B). This is consistent with a fact that the bare dimer reconstructs to stabilize itself energetically but the one-terminated dimer does not reconstruct at all. However, the difference is too small to distinguish from each other in experiments; therefore, this dimer must look as just a single spot in the NC-AFM images.

These characters are actually similar to scanning tun- 

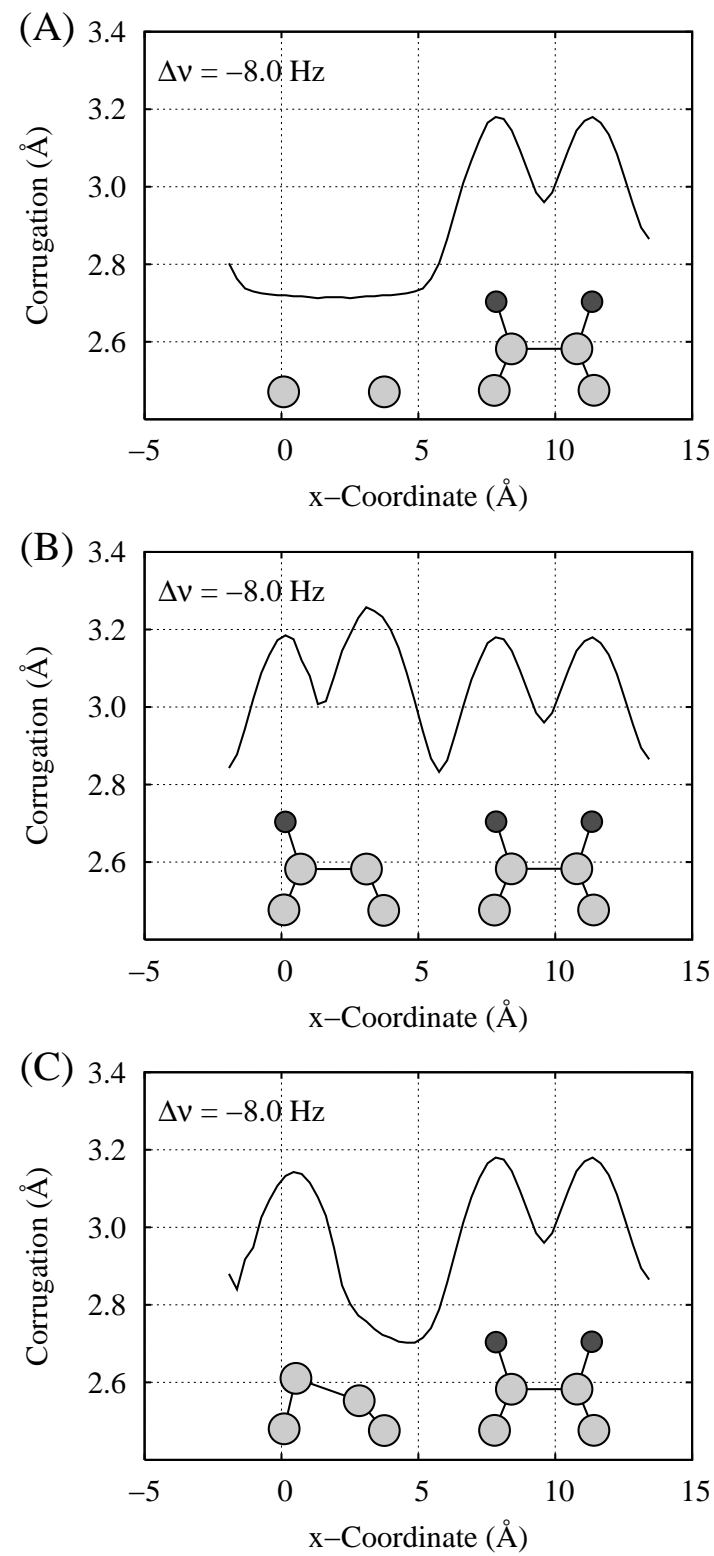

FIG. 3: NC-AFM line-profiles on the constant frequency shift mode. There are the missing dimer, the one-side-terminated dimer, and the bare dimer shown in (A), (B), and (C), respectively. Each irregular dimer is shown at the bottom together with the regular one.

neling microscopy (STM) [23-25]. However, the differences of the peaks are too small to distinguish the peaks like STM. The AFM signal becomes weak due to the additional distance from the tip apex at the vacancy site; moreover, the atomic force is the short-range interaction in contrast to the tunnel current in STM. Therefore, the tip-site dependence of the chemical activity easily cancels out the corrugations of the sample surfaces in AFM. Eventually, these irregular dimers show the curious behaviors.

\section{Thermal Effect}

The previous subsection concluded that the bare dimer causes a single bright spot in the experimental images. However, the bare dimer was freezing in this simulation, because the DFTB method assumed that the temperature was zero. When the bare dimer is observed at room temperature, it may induce the twin spots due to the thermal fluctuation, namely the flip-flop motion [26].

Hereupon, a rate of the flip-flop motion is compared with the rate of the cantilever vibration. The rate $R$ of the flip-flop motion under a temperature $T$ is given as follows:

$$
R=R_{0} \exp \left(-E_{B} / k_{B} T\right),
$$

where $R_{0}$ is the basic rate $\left(10^{11} \mathrm{~Hz}\right)$, and $k_{B}$ is the Boltzmann constant [26]. Besides, $E_{B}$ is the energy barrier required when the tilting bare dimer undergoes the flip-flop motion on the monohydride $\operatorname{Si}(001)$ surface. The energy barrier $E_{B}$ was $0.54 \mathrm{eV}$ in this study from the nudged elastic band (NEB) method on the DFTB method [27]. Substituting the value to the equation, the flip-flop motion rate with an order of $10^{4} \mathrm{~Hz}$ was obtained for a temperature $T$ of $300 \mathrm{~K}$. Therefore, the flip-flop motion is slower than the cantilever motion, because the frequency rate of the cantilever is an order of $10^{5} \mathrm{~Hz}$ (Table I) [7]. Consequently, the bare dimer must induce the single spot at the $300 \mathrm{~K}$.

\section{SUMMARY}

In this paper, the DFTB simulations were performed for NC-AFM line-profiling along three types of irregular dimers on the monohydride $\mathrm{Si}(001)$ surface. The three types of dimers were the one-side-terminated dimer, the bare dimer, and the missing dimer. The missing dimer induces no peak in the line-profile, which is a trivial result.

The curious behaviors were seen in the other two type dimers. The one-side-terminated dimer induces the twin peaks that are similar to the ones of the regular monohydride dimer, although a single hydrogen atom is on the irregular dimer. On the other hand, the bare dimer induces the single peak at the upper atom site only, although no hydrogen atom is on the dimer. The bare dimer has the flip-flop thermal fluctuation under finite temperature, but the single peak is kept even if the temperature arises up to room temperature.

These characters are similar to STM [23-25]; however, the differences of the peaks are too small to distinguish the peaks like STM. This is caused by that the signal is hardly detected due to the additional distance from the tip apex at the vacancy site, because the atomic force is the short-range interaction in contrast to the tunnel current in STM.
[1] F. J. Giessibl, Rev. Mod. Phys. 75, 949 (2003).

[2] F. J. Giessibl, Science 267, 68 (1995).

[3] N. Sasaki, S. Watanabe, and M. Tsukada, Phys. Rev.
Lett. 88, 046106 (2002).

[4] M. Harada and M. Tsukada, Phys. Rev. B 82, 035414 (2010). 
[5] L. Kantorovich and C. Hobbs, Phys. Rev. B 73, 245420 (2006).

[6] N. Sasaki, S. Watanabe, H. Aizawa, and M. Tsukada, Surf. Sci. 493, 188 (2001)

[7] S. Morita and Y. Sugawara, Jpn. J. Appl. Phys. 41, 4857 (2002).

[8] D. Sawada, T. Namikawa, M. Hiragaki, Y. Sugimoto, M. Abe, and S. Morita, Jpn. J. Appl. Phys. 47, 6085 (2008).

[9] A. Masago, S. Watanabe, K. Tagami, and M. Tsukada, Jpn. J. Appl. Phys. 48, 025506 (2009).

[10] A. Masago, S. Watanabe, K. Tagami, and M. Tsukada, e-J. Surf. Sci. Nanotech. 4, 197 (2006).

[11] N. Miura and M. Tsukada, Jpn. J. Appl. Phys. 41, 306 (2002).

[12] A. Natori, R. Nishiyama, and H. Yasunaga, Surf. Sci. 397, 71 (1998).

[13] H. N. Waltenburg and J. T. Yates Jr., Chem. Rev. 95, 1589 (1995).

[14] R. Perez, I. Stich, M. C. Payne, and K. Terakura, Phys. Rev. B 58, 10835 (1998).

[15] R. Perez, M. C. Payne, I. Stich, and K. Terakura, Phys.
Rev. Lett. 78, 678 (1997).

[16] M. Elstner, D. Porezag, G. Jungnickel, J. Elsner, M. Haugk, T. Frauenheim, S. Suhai, and G. Seifert, Phys. Rev. B 58, 7260 (1998).

[17] T. Frauenheim, F. Weich, T. Kohler, S. Uhlmann, D. Porezag, and G. Seifert, Phys. Rev. B 52, 11492 (1995).

[18] R. P. Feynman, Phys. Rev. B 56, 340 (1939).

[19] P. Ewald, Ann. Phys. 369, 253 (1921).

[20] N. Sasaki and M. Tsukada, Jpn. J. Appl. Phys. 37, L533 (1998).

[21] N. Sasaki and M. Tsukada, Appl. Phys. A 72, S39 (2001).

[22] J. Fritsch and P. Pavone, Surf. Sci. 344, 159 (1995).

[23] J. J. Boland, Phys. Rev. Lett. 67, 1539 (1991).

[24] X. Tong and R. A. Wolkow, Sci. Surf. 600, L199 (2006).

[25] C. H. Chen, K. D. Kepler, A. A. Gewirth, B. M. Ocko, and J. Wang, J. Phys. Chem. 97, 7290 (1993).

[26] D. Chen and J. J. Boland, Phys. Rev. B 65, 165336 (2002).

[27] D. Sheppard, R. Terrell, and G. Henkelman, J. Chem. Phys. 1128, 134106 (2008). 